\title{
Emergence of Time From Quantum Physics as Opposed to Abstraction of Time Components in Biopsychology
}

\author{
Franz Klaus Jansen \\ Independent Researcher
}

\begin{abstract}
Time is a highly complex concept, which is daily experienced as qualitative reality, utilized in science and classical physics as quantitative units, but denied by quantum physics. The time concept can be explained as emergence from the physics perspective, or as abstraction of time components, in the context of biopsychology, where quantitative time components are associated with the initial qualitative concept of time experience. Successive abstraction of the associated components renders the time concept more adaptable to different situations in both physics and daily life. The aspect of tense-with past, present, and future-positions time with respect to specific observers.However, as in physics, many experimental outcomes have to be grouped and individual; tenses must be excluded.The same is true for individual calendar references, which have to be replaced by time units. Newton's absolute time was replaced in Einstein's relativity theory by relative time. Whereas the aforementioned time concepts were based on waiting time corresponding to time flow characterized by waiting intervals between successive events, physics requires reference time stored on any kind of support, such as human memory or a magnetic deviceonly identifying time as its sources,. With support-fixed time, successive events are simultaneously accessible on the support, thus eliminating the waiting intervals. Support fixed time can be reduced to simple relations that appear timeless, and are more adapted to physical formalism.
\end{abstract}

Keywords: composite time, tense, time flow, time relations, quantum physics

\section{Introduction}

The concept of time can be defined from the physics or biopsychology perspective, which adopt opposite approaches. While time is an important factor for classical physics, it seems to be absent from quantum gravity, according to the Wheeler-DeWitt equation. Indeed, Kiefer (2009) observed,“this equation does not contain any classical time parameter $t$. The reason is that spacetime as such has disappeared in the same way as particle trajectories have disappeared in quantum mechanics” (p. 6). Only physical configurations remain, as if nothing ever happens in the universe.

Barbour (2009) justified this omission of time by stating, "In my view, had duration been properly studied in classical physics, its disappearance in the conjectured quantum universe would have appeared natural” (p. 1). Zeh (2000) similarly stated, "As we have known since Heisenberg's discovery, there are no paths in the quantum mechanical theory (therefore, in principle, no time-dependent pointer positions on clocks exist)” (p. 2)

Franz Klaus Jansen, MD, Ph.D., independent researcher, former: Biological Research in Immunology at the University of Düsseldorf, Germany, and later SANOFI, Pharmaceutical Company in Montpellier, France; now main research field: Philosophy of Science. 
(translation by the author). More recently, Rovelli (2009) observed, "In order to build a quantum theory of gravity the most effective strategy is therefore to forget the notion of time all together, and to define a quantum theory capable of predicting the possible correlations between partial observables” (p. 9). This approach is depicted in Figure 1. Although most physicists concur that time can and should be eliminated from quantum field theories, Smolin (2013) argued, "We need a new theory—one in which the reality of time is a central element” (p. 95).

Nevertheless, psychological time has to emerge in our brain, as Barbour (1994) indicated,

our memory is like a progress book, with snapshots taken every day and faithfully pasted in, one next to the other, the brightest and clearest from what we think is yesterday, the ones in the supposed past getting fainter and fainter. (p. 408)

An opposite approach to defining time relies on the experience of qualitative psychological time as duration without boundaries, according to Bergson (1922), since time is experienced every day by everybody. However, boundaries and other related factors are secondarily associated as different time components to the initial qualitative time concept (Jansen, 2018). Associated components can also be successively dissociated with the mental function of abstract reflection (Jansen, 2016). This increasing abstraction of associated components allows adapting qualitative psychological time to different situations in everyday life, classical physics, and finally timelessness in quantum gravity.

\section{Time Emergence From Physics as the Starting Point}

In physics, time is considered as an emergence from a timeless concept. According to Barbour (1994), "the passage of time is nothing but the difference between configurations, measured and parameterized in a more or less unique manner” (p. 407).

From a biopsychological perspective, Barbour's snapshots correspond to observations with sense organs. The obtained information is then stored in the observer's memory. The mental function of observation, based on elementary sensation, is time-consuming and imposed by the extra-mental environment (Jansen, 2016). However, after retrieval from the memory, a different mental function (abstract reflection) eliminates time components by successive abstractions, reducing them to relations. Hence, Barbour's snapshots represent time-consuming observations, which are stored in the memory. After retrieval and mental abstract reflection reduces them further to timeless configurations. Timelessness is therefore not physical reality but a mentally produced abstraction of physical reality.

This approach to understanding time can be illustrated with the example of purchasing a house near a railway, when the prospective buyer would be interested in the frequency of the passage of trains. Observation is time-consuming, as it involves waiting between two successive trains. This is defined as waiting time and corresponds to time flow. Based on these observations, the buyer may record passage of four trains per hour, storing this information in his/her memory. When the final result is retrieved from the memory, the time at which the four trains passed by the house is simultaneously accessible, no longer requiring waiting intervals between successive passages. This is defined as reference time, only identifying its source as earth time clock. However, earth time could have been replaced by any system with regular cyclical movements, such as moon phases, atomic time, or pulsar time. Reference time represents a conditional if-then time concept, for instance, if one loses the train departure, then one has to wait for a certain time.

In this case, time is no longer experienced as waiting time, but remains only mental potentiality. 
Nevertheless, without identification of the reference source, the correlation to an event can also be considered as a simple mathematical relation without any reliance on time, which then appears timeless.

In his attempt to justify timelessness of the universe, Rovelli (2009) proposed a mathematical approach describing the harmonic oscillation of a pendulum. The pendulum requires two measuring devices, one for the variable $\alpha$ representing the elongation of the pendulum and the other for the clock, the variable $\beta$ indicating the angle between the hand of the clock and the " 12 ". The first pair of $\alpha_{1}$ and $\beta_{1}$ variables appears simultaneously and is followed by successive pairs $\alpha_{2}$ and $\beta_{2}, \alpha_{3}$ and $\beta_{3}$, and so on (see Figure 1).

The measurement or observation process in this experiment requires reading of the $\left(\alpha_{i}, \beta_{i}\right)$ pairs from $\alpha_{1}$ and $\beta_{1}$ up to $\alpha_{n}$ and $\beta_{n}$. Each pair is read simultaneously, whereas the sequence of pairs is read successively and therefore requires waiting time intervals, which correspond to time flow. In contrast, if the measurement of all successive pairs of variables is finished and the data are stored on any kind of support, such as human memory, a piece of paper, or a computer, waiting time becomes support-fixed reference time (see Figure 1).

Fixed reference time is completely different from waiting time, since it no longer includes any waiting intervals between successive pairs of variables. They are now simultaneously accessible, like the frames of a film on a reel. As an example, a timetable indicating train departures is fixed reference time, since all departure times are simultaneously accessible. However, if one has no access to the timetable, to board the next train, one has to wait at the station until the next train is ready to depart. Fixed time without waiting intervals thus represents a reduction of time flow. The fixation procedure eliminates waiting time in science as well as in everyday life and reduces it to source-identified reference time.

Rovelli (2009) argued that reference time can be reduced to timeless relations:

we can find mathematical laws characterizing sequences of events.... Call an unparametrized curve $\gamma$ in $\mathrm{C}$ a motion of the system. Perform a sequence of measurements of pairs $(\alpha, \beta)$, and find that the points representing the measured pairs sit on a motion $\gamma$. Then we say that $\gamma$ is a physical motion. We express a motion as a relation in C. (p. 4)

In Rovelli's sequence of measurements, waiting time between successive pairs of variables is implicitly included, whereas the points on a curve denote fixed time, which is simultaneously accessible without waiting intervals. The curve is then expressed as a simple relation, in which the reference source to which time is linked is eliminated, rendering the relation timeless. Does this mean that time is completely eliminated?

One may compare Rovelli's example of a pendulum to the problem of inflation in economics. An annual salary indexed to the inflation rate payable in US dollars may be treated as the variable $\alpha$, while $\beta$ can denote the number of years. A curve linking these variables will indicate annual variation in salary, where each $(\alpha, \beta)$ pair will be followed by successive pairs, until the final measurement, denoted by $\alpha_{n}$ and $\beta_{n}$. If the curve can be described by a simple mathematical relation, it may permit inflation to be precisely determined, without any knowledge of the underlying currency. However, without the knowledge of currency, important information on the country to which the inflation relates would be lost. Calculated with dollars, the inflation would relate to the US, while Yuan would point to China. If the currency and therefore the corresponding country remain unknown, the calculated inflation is taken out of its context and becomes meaningless, since it represents only a part of the required information.

The reduction of correlations between source-identified entities, for instance currencies, to simple mathematical relations, renders the calculated information contextless, since it loses source identity. However, contextless information represents only a fraction of the expected meaningful information. Mathematical 
relations leading to timeless configurations of the universe have similarly lost their source identification and can be considered contextless. When mathematical relations are once again considered in the whole context with their source identity, time reappears, indicating that it was merely hidden in contextless mathematical equations.

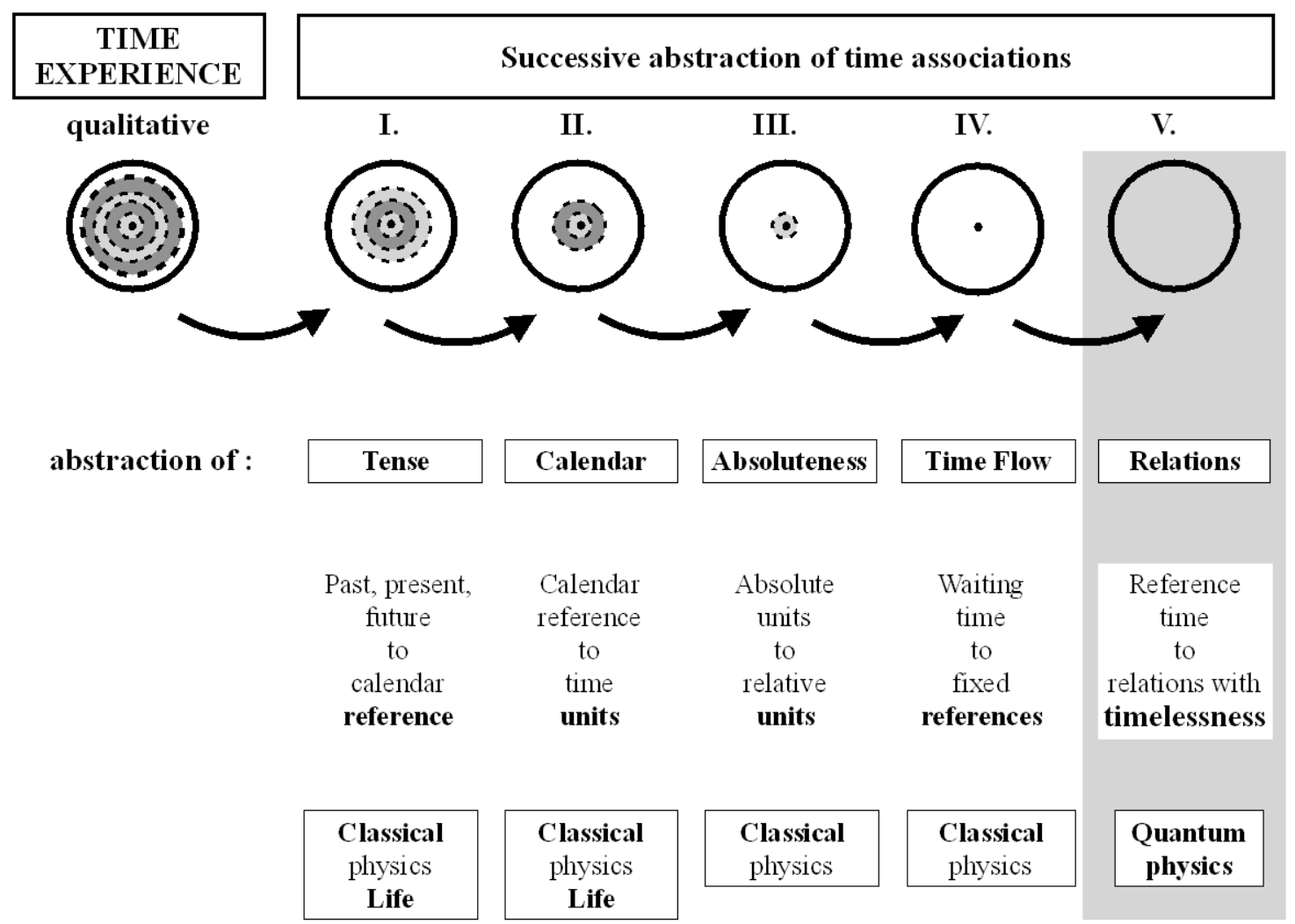

Figure 1. Successive abstraction of time components.

Experienced time is associated with multiple time components, which can also be dissociated by successive abstraction. Different abstraction steps render the complex composite time more adaptable to different situations in life and classical physics. Finally, it is reduced to timelessness in quantum field theories.

\section{Time Experience in Biopsychology as the Starting Point}

Before the notion of time can be applied in physics, it is psychologically experienced as duration, which cannot be divided and measured, according to Bergson (1922). Nevertheless, primarily experienced time can be secondarily associated with measurable time units, for instance, by comparison with recurring motions, like a clock or behavior of other repetitive cyclical systems. The time concept is usually associated with past, present, and future, as well as with calendar references, time intervals, and absolute or relative time units. Moreover, all these associations can also be dissociated by the mental function of abstract reflection (Jansen, 2016) from the experienced psychological time concept, finally reducing time in quantum field theories to simple mathematical relations, which are timeless (Jansen, 2018). 


\section{Abstraction of Tense}

Past, present, and future are commonly used terms to describe events in everyday life. However, they only have a specific meaning with respect to an observer:

The present is experienced through the interaction of active sense organs with extra-mental reality, which imposes waiting time (see Figure 1).

1. The past is a recollection of recorded past events, which are stored in one's memory or other storage media but no longer need active sense organs.

2. The future is not yet realized and remains uncertain potentiality of anticipated events, which may or may never manifest in reality.

Without attaching these specific meanings, the notions of past, present, and future would have lost their essential sense. Consequently, tense is related to an observer, who interprets it with respect to his/her actual present.

McTaggert (1908) proposed an alternative definition of past, present, and future, which does not require any reference to an observer, using the death of Queen Anne as an example. He noted, "It began by being a future event. It became every moment an event in the nearer future. At last it was present. Then it became past" (p. 3). Since such an event cannot simultaneously possess three contrasting properties concerning time (i.e., past, present, and future), McTaggert considered time as unreal.

However, McTaggert's definition of tense corresponds to a retrospective perspective, since in his definition, any future event is already known and therefore lies in the past, when the date of Queen Anne's death and all circumstances of her death were well-known. In contrast, the future is generally considered in a prospective perspective and in relation to an observer, since future does not yet exist, as all individuals live in the present. With respect to an observer living during the time of Queen Anne's rule, the date of her death is not yet known and, in his present, the conditions of her death are not yet publicly available. Thus, tense becomes only meaningful with respect to one or multiple observers.

In physics, an experiment is performed in the present of the individual conducting the measurements, and thereafter becomes an event that has taken place in the past, whereas future experiments are not yet realized. Since physical laws cannot be based on only one experiment that is conducted in the present, they require repetition of multiple experiments, all of which will have occurred in the past by the time conclusive evidence is collated. The grouping of experimental outcomes cannot take into account the individual past, present, or future, which are therefore eliminated. This is in agreement with Einstein's (1955) remark that "the separation between past, present and future has only the importance of an admittedly tenacious illusion” (p. 1).

\section{Abstraction of Calendar References to Time Units}

Experiments performed in the present are linked to common calendar references, such as the Gregorian calendar, which allow positioning an event in a precise time frame (see Figure 1). However, as with tense, grouping of experimental outcomes performed at different calendar times can no longer be related to individual calendar references. Grouping thereby requires quantitative time intervals calculated in units of clock time, generally expressed in hours, minutes, or seconds, based on Earth'srevolution.

Quantitative time units are necessary in physics, as well as in daily life. Measurement of a time interval expressed in time units of a recent car trip allows the time necessary to complete similar future car trips to be estimated. 


\section{Abstraction of Absolute to Relative Time Units}

Newton considered absolute time, whereas it had to be replaced by relative time in Einstein's relativity theory (Miller, 1998), which is dependent on the speed of an observer with respect to the speed of light (see Figure 1). Since light travels at constant speed, time passes more slowly when an observer travels at a velocity approaching the speed of light. In normal life, events occur at much slower speeds, and no distinction between absolute and relative time is generally experienced. Newton's and Einstein's time concepts are experienced as waiting time corresponding to time flow; nevertheless, they can be further reduced to fixed time.

\section{Abstraction of Waiting Time Flow to Support-Fixed Reference Time}

In daily life, both waiting and fixed time also exist. Time flow is the normal time experience requiring waiting intervals between events, whereas with fixed reference time, stored in the memory and then retrieved, all events are simultaneously accessible without waiting intervals.

The key difference between time flow and fixed reference time can be best illustrated with a film (Jansen, 2018). During registration of a bike race on a film, time cannot be changed by accelerating or slowing down the race. Yet, once the race has been stored, the original time flow had been fixed on the support of the camera. When the film with fixed time is projected, the bicycle race can be accelerated or slowed down by manipulating the support on which it is fixed. Thus, while the recorded waiting time has not been changed, if the movement of the support is modified, an artificial time is created, which might or might not correspond to the original time flow during the race. Accurate restoration of the original time flow can only be obtained after calibrating the movement of the support with a clock present during registration of the original time.

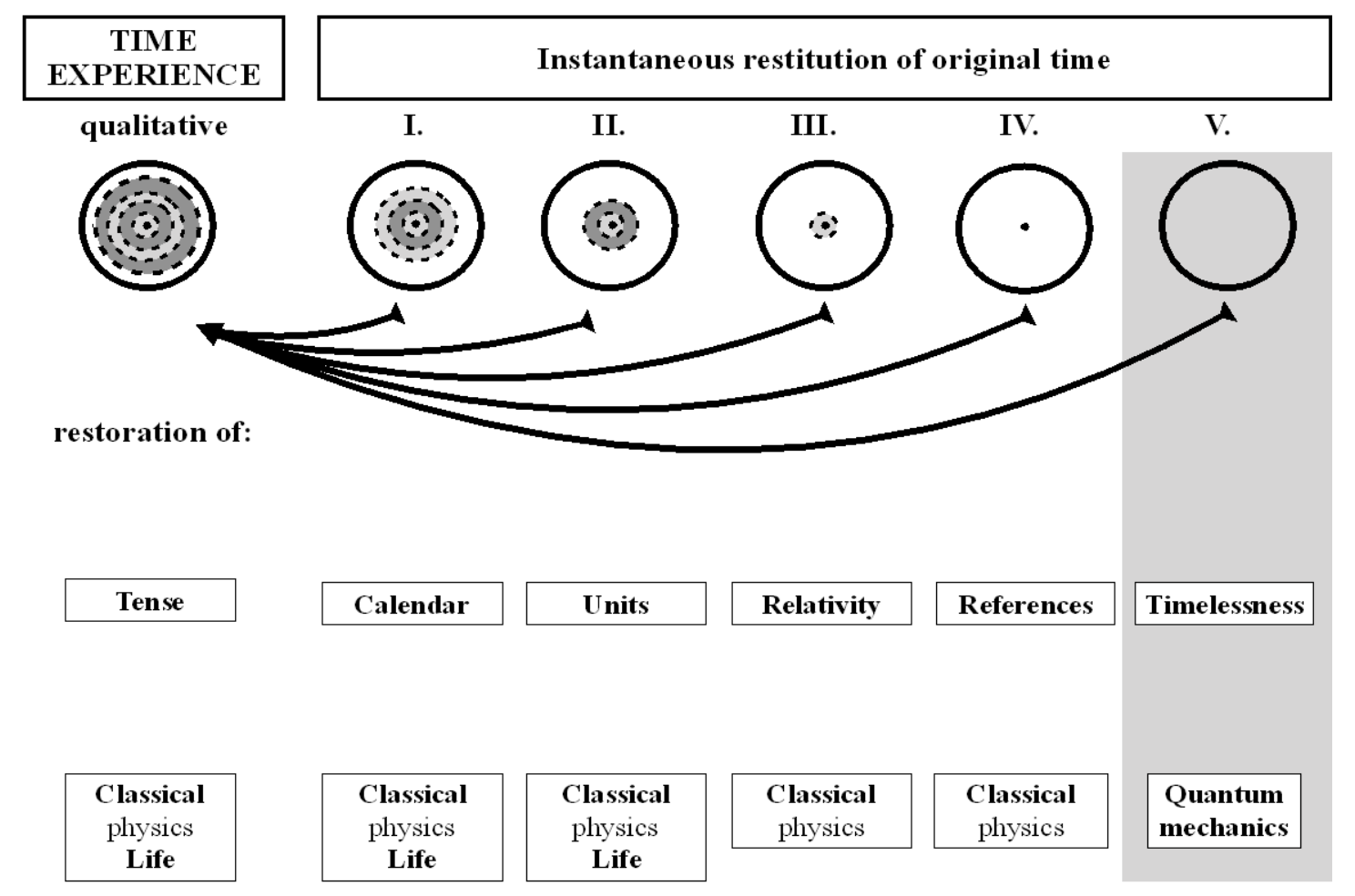

Figure 2. Time restitution by experimental verification. 
Each layer of time reduction by abstraction provides a better adaptation to certain circumstances in life and in physical formalism. Since the predictions based on physical formalism have to be verified through new experiments in order to be deemed reliable, experiential time with all its components instantly reappears, proving that successive abstractions were merely mental reflections, rather than observable physical facts.

\section{Abstraction of Mathematical Correlations to Timelessness}

In daily life, timelessness can be experienced in certain situations, such as during meditation. By closing the eyes to inhibit vision, by remaining in a quiet environment to limit audition, and by avoiding any movements and concentrating on the present only, one can reach a state that is felt as timelessness.

Psychological time experienced as duration (Bergson, 1922), including all its different associated components, was adapted through five successive abstractions to different physical situations and finally led to timelessness. Some physicists argue that time completely disappears in the physical realm; consequently, the perception of time gained through everyday experiences is merely a psychological illusion. Nevertheless, experienced time with all its components can be easily restituted, since it is merely hidden due to successive abstractions of its components.

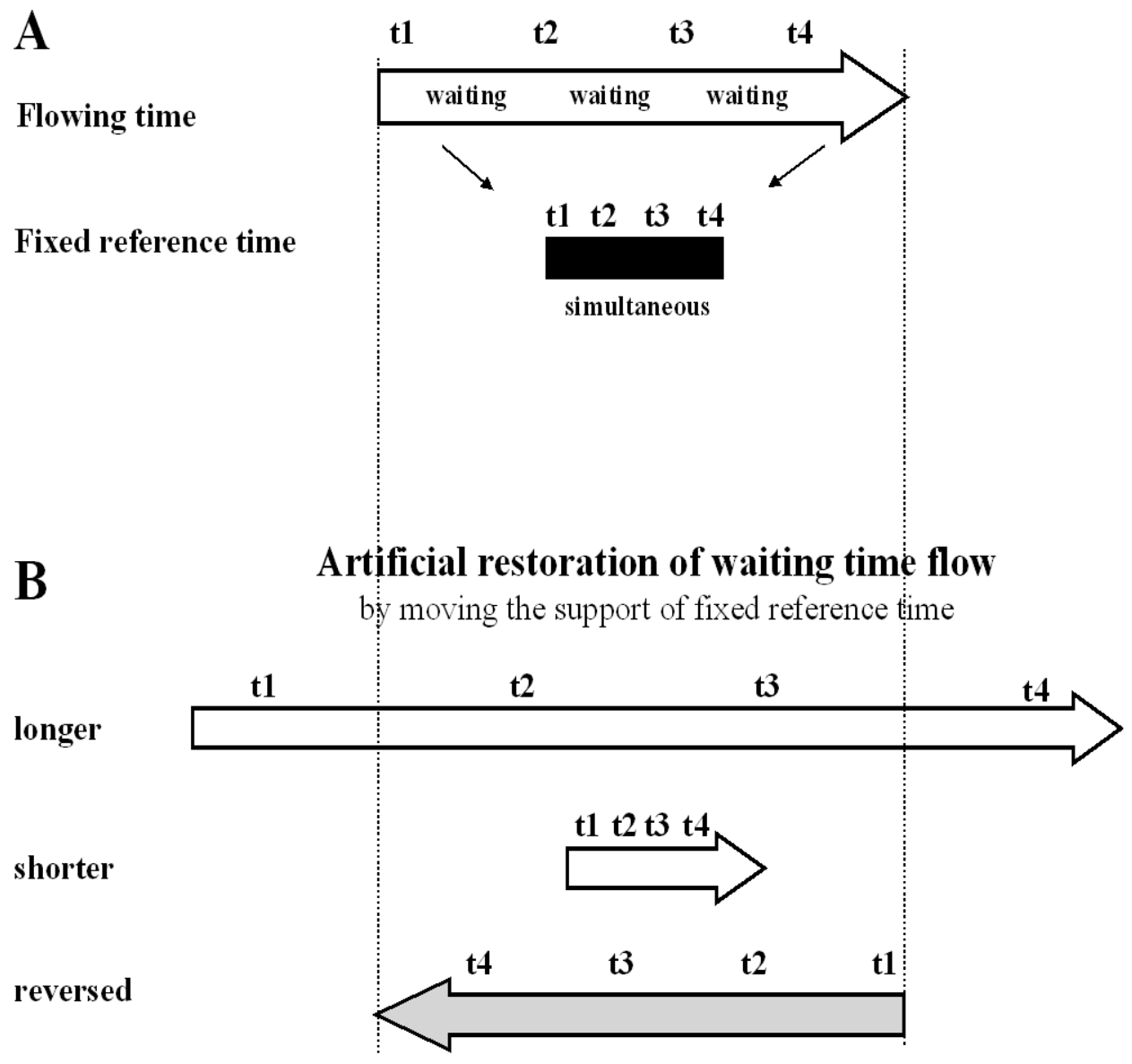

Figure 3. Original flowing time and fixed reference time.

(A) Flowing time experience is characterized by waiting intervals between successive events, whereas fixed time traces are simultaneously accessible on a specific support. 
(B) Flowing time can be artificially restored from fixed reference time by manipulating the support and can become longer, shorter, or even reversed. Only restored time calibrated with a clock resembles the original flowing time.

\section{Restitution of Time Experience in Biopsychology}

Physical laws are established to quantify and explain natural phenomena, and thus predict their future behaviors and outcomes. When such predictions pertain to regular behaviors, such as a ball rolling down an inclined plane in Galilee's experiments (Straulino, 2008), they can be made with a high degree of certainty. Conversely, in quantum mechanics, predictions relate to irregular outcomes, and are thus limited to probability estimations.

Since physical formalism allows predicting future outcomes, its reliability can be experimentally validated. Predictions should correspond to future realizations of experimental outcomes and thereby restitute all abstracted time components (see Figure 2). In quantum mechanical verification experiments, timelessness is substituted by reference time. When Rovelli's mathematical relations between pairs of variables without time consideration are realized in new experiments, once again, waiting intervals between successive variables are introduced, and thereby restitute time flow. Time intervals are once more linked to calendar time and tense becomes meaningful with respect to the experimentalist.

This example confirms that, when predicted experiments are realized in practice, all prior abstractions are abandoned, which proves that abstractions imposed by physical formalism are pure mental reflections, not observable physical reality.

Barbour's (2009) claimed "I think it is entirely possible-indeed likely-that time as such plays no role in the universe" (p. 8) could be differently interpreted from a biopsychological perspective as "time plays no role in the physical formalism of the universe". Kiefer's (2009) timeless world could similarly be conceived only as "a timeless physical formalism of the world". Finally, Rovelli’s (2009) argument that we should simply "forget time” (p. 1) should also be limited to physical formalism, as it can never eliminate the reality of waiting time experienced in everyday life. The recovery of time in physics proceeds in different steps from timelessness to biopsychological time flow.

\section{Timeless Equations Merely Obscure Reference Time}

Timelessness can emerge if reference time identified by clock or other cyclical system is reduced to simple mathematical relations. Such elimination of any source identity can be considered as privation of a larger context required for a better understanding of the significance of a mathematical relation. In the context in which reference sources are identified, time once again reappears. Thus, time is inevitably present in the whole context, and is merely obscured via contextless mathematical relations. This prompts the question of whether contextless mathematical equations describing only partial aspects of a whole are a trustworthy representation of physical reality.

\section{Source-Identified Reference Time Corresponding to Clock Time}

Reference time with identified sources can be understood as a comparison of observed physical facts to independent clocks, which can be of various types. Successive events fixed on any kind of support are simultaneously accessible to an observer and no longer require waiting time intervals between individual events, but nonetheless reference their context. In a film, multiple frames represent multiple snapshots, each of which 
pertains to a different point in time. Snapshots are simultaneously present on the reel, although they were registered sequentially.

Rovelli's (2009) registered variables, recorded on a paper or a screen, are also simultaneously present on their support, thus eliminating the waiting intervals between observations of pairs of variables.

Similar to a film, physical formalism can also be considered as a support for fixed time $(t)$, which depends on mathematical rules. The sequence of time intervals, such as $t_{1}, t_{2}, t_{3}, \ldots t_{n}$, stored on a support like a paper or a screen is simultaneously accessible to an observer, since there are no waiting intervals between any of the variables.

\section{Restoration of Fixed Reference Time to Waiting Time}

Reference time fixed on a particular support is entirely dependent on the characteristics of that support. Thus, waiting time can be restored by manipulating the support (see Figure 3). However, this is still an artifact, since the stored events can be made to occur more rapidly or more slowly than those that were originally registered.

Original flowing time can be precisely restored only if the movement of the support is calibrated to coincide exactly with the passage of time in reality, as measured by a clock. Exact correspondence between the recreated time flow and that in the original event is essential for the moving support to reproduce the original time with fidelity.

Waiting time signifies separation of successive events by waiting intervals, since they occur sequentially. The successive appearance of events contributes to the impression of time flow, similar to Heraclitus's assertion that everything flows and nothing abides (Savitt, 2017, introduction) (see Figure 2).

\section{Non-experiential Reference Time}

Fixed reference time cannot be restored to waiting time if time intervals exceed the human capacity of time experience. Time intervals can be too long, such as those in archeology or cosmology, or too short to be comparable to any everyday process (such as a tenth of a second). Nevertheless, they are commonly encountered in physics, but cannot be experienced by humans.

In archeology, one can find superposed sediment layers in caves, indicating different time periods of a distant past. Without any trace of organic radioactive substances, like C14, in the corresponding layers that could be used to estimate passage of time in distant past by radioactive decay, precise dating would be impossible.

\section{Time Reversal in Physics and Life}

Incorrect manipulation of the support on which past events are stored can create an artificial time reversal, for instance in a film showing a diver jumping from a springboard into the water below, and then making the diver fly back up to the springboard in the reversed time direction. Since time is fixed on its support (in this case a film), changing the direction of the support movement would produce artificial time reversal, creating the illusion that time could be generally reversed, which is clearly not the case.

Physical formalism can also be considered as a support for fixed reference time, which is grounded in mathematical laws. In Encyclopedia Britannica (2018), time reversal is defined as follows, "in physics, mathematical operation of replacing the expression for time with its negative in formulas or equations so that they describe an event in which time runs backward", which implies "that the flow of time does not have any naturally preferred direction”. Atmanspacher and Filk (2012) argued, "It is important to realize, however, that 
there are no a priori reasons to select one of the two temporal directions at the expense of the other. Such a selection has to be based on additional arguments” (p. 89).

Mathematical formalism is the support for fixed time in physics, which permits time to change direction simply by considering its negative value in the corresponding formalism without affecting the validity of physical laws. Physicists consider mathematics as the only means of describing natural phenomena, due to which it should be possible for time to run forwards as well as backwards. However, as Stewart (2017), a renowned mathematician, stated, “There is strong consensus that mathematics isn't reality, it just resembles reality in a useful way” (p. 129).

In human memory, past events and their fixed time traces are physiologically stored without any references to their sequential order. Thus, it is possible to recall these events in any order, creating an illusion of time flowing in both directions, as in mathematics. In daily life, the precise succession of events can also be forgotten, permitting the question of, in a reversed time direction, what would precede a particular event. In science, to establish the cause of an observed effect, it is also necessary to proceed in the reversed time direction. Such actions would correspond to the negative time direction in mathematics.

In general, time fixed on any kind of support can be modified in multiple ways, such as by accelerating, slowing down, excluding time intervals, or reversing the time arrow. However, none of these modifications is possible during registration of time flow with waiting intervals between successive events, as was demonstrated with a film.

\section{Conclusion}

Time is often considered as a unique concept, which can lead to interpretation issues when referring to time in different domains, such as physical formalism and daily life. Time can be interpreted from the perspective of quantum physics as a psychological emergence or illusion. However, time experienced in everyday life can also be explained from a biopsychological perspective. In the latter case, time is considered a complex phenomenon, comprising of different components, starting with Bergson's duration without boundaries. It is associated with past, present, future, calendar references, quantitative time units, absolute and relative time, waiting time flow, and reference time. The dissociation of these time components through successive abstraction leads to a reduction of time components.

Qualitative experience of time is the starting point of a highly complex time concept in biopsychology. In order to be adapted to different situations, the complexity of the time concept has to be reduced by successive abstraction of its components. Past, present, and future have different meanings that would be incomprehensible without considering the role of an observer. Physical laws are based on the findings yielded by repeated experiments that have been conducted in the past. By grouping the outcomes of identical experiments that have been performed over a period of time the temporal relationship between individual observations becomes meaningless and has to be abstracted to be adapted to physical formalism. Therefore, past, present, and future have no meaning in physics (Einstein, 1955).

Physical experiments are realized within a common reference frame of calendar time. Consequently, by grouping multiple experimental outcomes, their individual calendar time properties are eliminated. Therefore, calendar time properties have to be abstracted in favor of time units that are completely independent of calendar time references. 
Newton considered time intervals as intrinsically linked to absolute time, whereas Einstein's relativity theory imposed relative time, necessitating that absolute time be abstracted and replaced by relative time.

Although relative time still represents time flow, which requires waiting intervals between successive events, support-fixed reference time implies source identity and does not require waiting intervals between events, as these are now simultaneously accessible. After elimination of its source identity, fixed reference time becomes a simple relation in physics, which can be perceived as timelessness.

The described successive abstractions are only mental operations that render the time concept more adapted to specific physical contexts or life circumstances. Therefore, abstractions of time components are virtual and do not represent elimination of experienced waiting time corresponding to time flow, which remains reality.

Mathematical formalism is utilized to describe physical laws, which are treated as immutable. Thus, it allows future experimental outcomes to be predicted with relative certainty. Using mathematical formalism, reliability of previously established physical laws can be tested by repeating relevant experiments and checking if their outcomes correspond to the predictions based on laws. However, when verification experiments are conducted in quantum physics, all time components reappear, such as time units, waiting time, and tense. In other words, time components are only mentally abstracted in mathematical representations and are automatically restored when verifying them through experiments in physical reality.

Waiting time flow and support-fixed reference time-as two essential time components—are separated by a great gap. During event registration with a camera, waiting time does not allow even a slightest change, whereas reference time fixed on the film will permit multiple changes during restoration by projection, since it is now only dependent on the manipulation of the support. Uncalibrated restorations are pure artifacts, since time can be rendered to pass more rapidly or slowly, and it is even possible to reverse the time arrow. The original waiting time can only be restored when the film on which it was fixed is calibrated with a clock that was present during the registration process.

Physicists claim that an invariant affine time group determines the nature of time, which therefore allows time reversal. Such mental reflections are possible in mathematical formalism and during retrieval of memory-stored events, but they are strictly limited to support-fixed reference time. They can never eliminate the existence of waiting time flow, experienced in everyday life, which is completely incompatible with the notion of time reversal.

\section{References}

Atmanspacher, H., \& Filk, T. (2012). Determinism, causation, prediction, and the affine time group. Journal of Consciousness Studies, 19(5-6), 75-94.

Barbour, J. B. (1994). The emergence of time and its arrow from timelessness. In J. J. Halliwell, J. Perez-Mercader, W. H. Zurek (Eds.), Physical origins of time asymmetry (pp. 405-414). New York: Cambridge University Press.

Barbour, J. B.(2009). The nature of time. Retrieved 15/01/2018 from https://arxiv.org/pdf/0903.3489.pdf

Bergson, H. (1922). Duréeetsimultanéité. À propos de la théorie d' Einstein.Alcan, Paris: Librairie Felix.Retrieved15/01/2018fromhttp://classiques.uqac.ca/classiques/bergson_henri/duree_simultaneite/duree_et_simultaneite. pdf

Einstein, A. (1955). Letter to the Besso family. Retrieved 15/01/2018 from http://www.christies.com/features/Einstein-letters-to-Michele-Besso-8422-1.aspx Encyclopedia Britannica. (2018). Retrieved 15/9/2018 from https://www.britannica.com/science/time-reversal 
Jansen, F. K. (2016). Elementary sensation primacy creating a first to third person gap of consciousness. International Journal Psychology Research, 10, 87-111.

Jansen, F. K. (2018). Composite time concept for quantum mechanics and bio-psychology. Philosophy Study, 8(2), 49-66.

Kiefer, C. (2009). Does time exist in quantum gravity? Retrieved 15/01/2018 from https://fqxi.org/data/essay-contest-files/Kiefer_fqx.pdf

McTaggert, J. E. (1908). The unreality of time. Mind: A Quarterly Review of Psychology and Philosophy, 17, 456-473.

Miller, A. I. (1998). Albert Einstein's special theory of relativity: Emergence (1905) and early interpretation (1905-1911). New York: Springer.

Rovelli, C. (2009). Forget time. Retrieved 20/9/2018 from https://arXiv:0903.3832

Savitt, S. (2017). Being and becoming in modern physics. Stanford Encyclopedia of Philosophy. Retrieved 15/01/2018 from https://plato.stanford.edu/entries/spacetime-bebecome/

Smolin, L. (2013). Time reborn: From the crisis in physics to the future of the universe. New York: Spin Networks

Stewart, I. (2017). Infinity: A very short introduction. New York: Oxford University Press.

Straulino, S. (2008). Reconstruction of Galileo Galilei’s experiment: The inclined plane. Physics Education, 43(3), $316-321$.

Zeh, D. H. (2000). Was heisst: esgibtkeineZeit? (Which means: there is no time?). Retrieved 26/07/2018 from http://www.zeh-hd.de 